\title{
INSTRUMENTAÇÃO E MONTAGEM DE MESA CIRÚRGICA PARA SEPTOPLASTIA COM TURBINECTOMIA
}

\author{
INSTRUMENTATION AND TABLE SETUP FOR SEPTOPLASTY SURGERY \\ WITH TURBINECTOMY
}

Henrique de Paula Bedaque1; Lucas Marinho Vasconcelos ${ }^{1}$; Deborah Carla Santos Gibson'; Halan Araújo Santos'; Lidiane Maria de Brito Macedo Ferreira²; Maria Luísa Nobre Medeiros e Silva Guimarães ${ }^{3}$

1. Médico, Residente de Otorrinolaringologia. Universidade Federal do Rio Grande do Norte (UFRN). Natal-RN. Brasil.

2. Médica otorrinolaringologista. Professora adjunta do departamento de cirurgia da UFRN. Natal-RN. Brasil.

3. Médica otorrinolaringologista, programa de pós-graduação em Ciências da Saúde da Universidade Federal do Rio Grande do Norte. Natal-RN. Brasil.

Study performed at Otorhinolaryngology and Head and Neck Service at Onofre Lopes University Hospital, Brazil.

Financial support: None.

Conflicts of interest: None.

Corresponding author: Otorhinolaryngology and Head and Neck Service at Onofre Lopes University Hospital - Av. Nilo Peçanha, 620 - Petrópolis, Natal - RN CEP: 59012-300.

E-mail: orlhuol@gmail.com.

Submitted: may 12; accepted after revision, sep 16, 2021.

\section{RESUMO}

Introdução: $\mathrm{O}$ ato cirúrgico de sucesso depende de um conjunto de processos e habilidades; dentre eles, instrumentos cirúrgicos adequados em uma disposição que traga facilidade e agilidade ao procedimento são parte importante do ato cirúrgico. Objetivos: o presente artigo busca revisar a nomenclatura de instrumentos cirúrgicos usuais na cirurgia de septoplastia e turbinectomia, bem como propor uma organização sistemática de mesa cirúrgica. Métodos: Utilizando imagens de instrumentos reais de forma individual e em suas posições na mesa de instrumentação, busca-se tornar didático o ensino de boas práticas de instrumentação. Conclusão: O conhecimento sobre a nomenclatura dos instrumentos cirúrgicos e sua adequada organização para o ato operatório podem ser facilitadores para um procedimento de sucesso.

Descritores: Septoplastia. Instrumentos Cirúrgicos. Ensino. 


\section{ABSTRACT}

Introduction: The success of surgical procedures depends on a set of processes and skills; among them, appropriate surgical instruments and a provision that brings ease and agility to the procedure are an important part of surgery. Objectives: this article seeks to review the nomenclature of usual surgical instruments in septoplasty and turbinectomy surgery, as well as propose a systematic organization of the operating table. Methods: Using images of real instruments individually and in their positions on the instrumentation table, it is sought to make teaching good instrumentation practices didactic. Conclusion: Knowledge about the nomenclature of surgical instruments and their proper organization for the surgery can be a facilitator for a successful procedure.

Keywords: Septoplasty. Surgical Instruments. Teaching.

\section{INTRODUÇÃO}

O septo nasal é uma parede óssea, cartilaginosa e mucosa que separa cada uma das cavidades nasais medialmente. Idealmente, a estrutura deverá se comportar de forma reta, porém decorrente das grandes variações anatômicas normais e de traumas de face o desvio de septo nasal é uma das alterações mais comuns da otorrinolaringologia ${ }^{1,2}$.

Um septo nasal desviado ou com alguma convexidade leva a uma mudança do fluxo aéreo no meato nasal ${ }^{1}$. Dessa forma, poderemos ter um menor espaço de perviedade aérea no meato nasal, o que leva a sintomas de obstrução nasal, principalmente pelo contato da concha nasal inferior com a parede septal $\left.\right|^{2,3}$.

Além disso, quando lidamos com o lado convexo do septo teremos um maior espaço meatal que normalmente leva a formação de uma concha nasal inferior vicariante, isto é, hipertrófica, edemaciada e sendo mais um fator etiológico para a queixa de obstrução nasal de um paciente com desvio septal ${ }^{2}$.

Em soma, pacientes com rinite alérgica ou rinossinusite crônica tem o desvio septal mais um fator de piora para o sintoma obstrutivo, piorando a qualidade de vida desses pacientes ${ }^{3}$. Nessas situações, a cirurgia de correção do desvio septal com a remoção parcial da concha nasal inferior pode ser muito bem indicada no tratamento de pacientes sintomáticos ${ }^{4}$.

A septoplastia com turbinectomia é umas das cirurgias mais realizadas na otorrinolaringologia e tem por princípio a retirada da região septal desviada e remoção parcial da concha nasal inferior'. Assim, restaura-se um fluxo aéreo mais adequado, reduzindo as queixas de obstrução nasal do paciente. O conhecimento técnico sobre a realização deste procedimento é de suma importância para o sucesso da intervenção ${ }^{5,6,7}$. 
Instrumentação e montagem de mesa cirúrgica para septoplastia com turbinectomia Bedaque HP, et al

Atualmente, devemos ampliar o conceito de técnica cirúrgica para além dos passos operatórios, pois uma boa técnica cirúrgica depende de uma boa interação com a equipe, com um bom instrumental e boa anestesia. Esse conceito se mostra importante no sentido de participar ativamente dos protocolos de cirurgia segura, na sua etapa inicial quando se chega o instrumental disponível ${ }^{8}$.

Ademais, o uso padronizado de uma mesa de instrumentalização proporciona um ambiente mais reprodutível e educativo para a formação de um residente na área cirúrgica. Estudos também mostram que a revisão sobre os instrumentais necessários para o ato cirúrgico pode reduzir custos, na medida em que se evitam gastos com esterilização de material não utilizado ${ }^{9}$.

Dessa forma, entendemos que a montagem de uma mesa de instrumentação organizada, com bom instrumental e diante de um assistente com domínio dos processos da técnica cirúrgica podem fazer uma grande diferença na fluidez do ato operatório. Assim, descreveremos a montagem de mesa cirúrgica para uma das técnicas de septoplastia com turbinectomia, com o objetivo de auxiliar em uma boa instrumentação.

\section{MATERIAIS}

Inicialmente deve-se dominar o nome e a função de cada um dos instrumentos cirúrgicos disponíveis na caixa cirúrgica. Esse ponto é essencial para facilitar o diálogo do cirurgião com a sua equipe, tornar o processo de instrumentalização mais dinâmica e fluida.

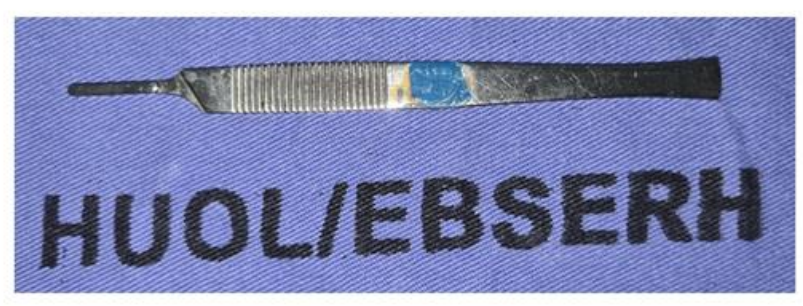

Cabo de Bisturi $\mathrm{N}^{\circ} 3$

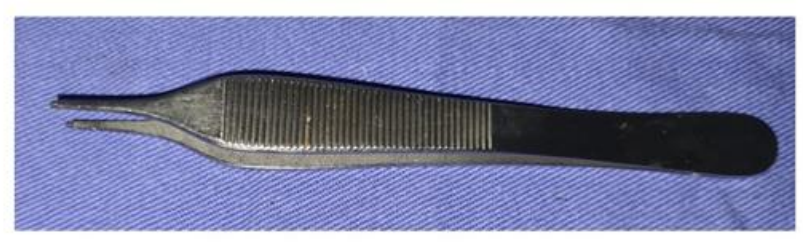

Pinça Adson com dente

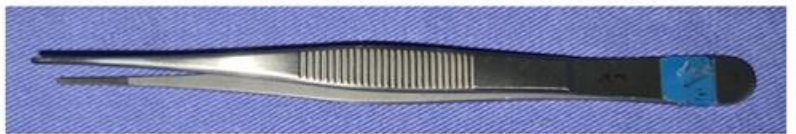

Pinça Anatômica

Fonte: Unidade de Cabeça e Pescoço HUOL/EBSERH.

Figura 1 - Instrumentais Cirúrgicos 1

O cabo de bisturi (Figura 01) é um suporte para o melhor manuseio da lâmina de bisturi utilizado para o corte a frio de epitélios e mucosas. Na realização da septoplastia, 
Instrumentação e montagem de mesa cirúrgica para septoplastia com turbinectomia Bedaque HP, et al

o corte da mucosa nasal é uma das etapas mais iniciais e a lâmina mais utilizada é a de número 15. Além disso, na figura acima vemos a pinça de Adson com dente e a pinça anatômica sem dente, o racional é ter pinças finas com e sem dentes para o adequado manuseio das mucosas em diferentes momentos cirúrgicos.

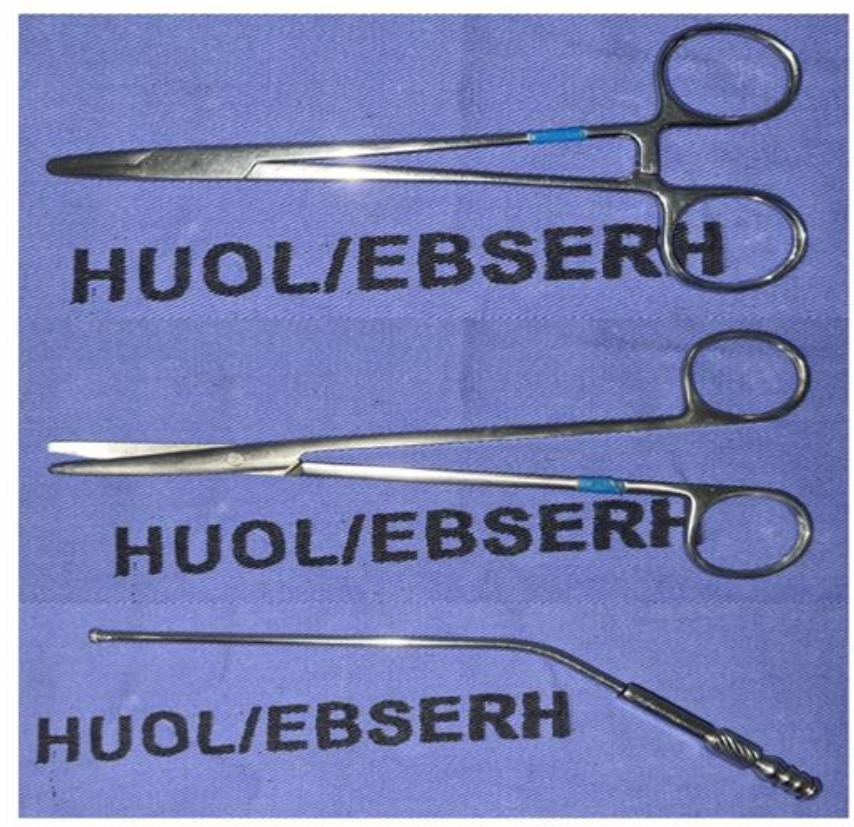

Porta Agulha Mayo Hegar

Tesoura de Mayo Reta

Fonte: Unidade de Cabeça e Pescoço HUOL/EBSERH.

Figura 2 - Instrumental Cirúrgico 2

O porta agulhas de Mayo Hegar (Figura 02) é uma ferramenta preciosa para facilitar o processo de sutura, etapas finais da septoplastia. É tecnicamente adequada a utilização de fios absorvíveis para a sutura das mucosas e para a aproximação das mucosas septais dissecadas, como catgut simples, já se a técnica envolver a colocação de splint nasal (anteparo situado em cada uma das fossas nasais para manter as mucosas septais unidas e sem contato com a região friável das conchas nasais) utilizamos de fios não absorvíveis, como o nylon na sutura.

Na figura acima mostramos a tesoura de Mayo reta, entretanto a curva também será tecnicamente adequada, as quais utilizamos para cortar os pontos de sutura e demais procedimentos de cortes necessários. O aspirador nasal atraumático é utilizado durante todo o procedimento para a aspiração de secreções e de sangue que por ventura estejam na região do meato nasal ou na rinofaringe. A manutenção de um campo operatório limpo é essencial para a adequada execução técnica da cirurgia. 
Instrumentação e montagem de mesa cirúrgica para septoplastia com turbinectomia

Bedaque HP, et al

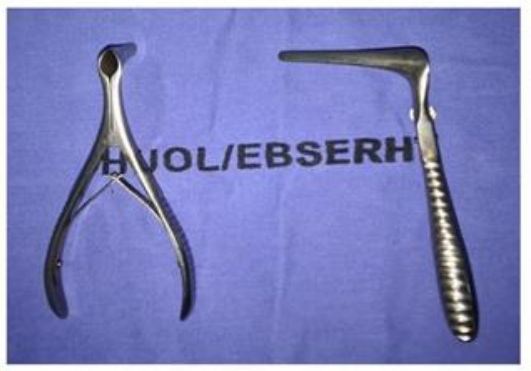

Espéculo de Killian

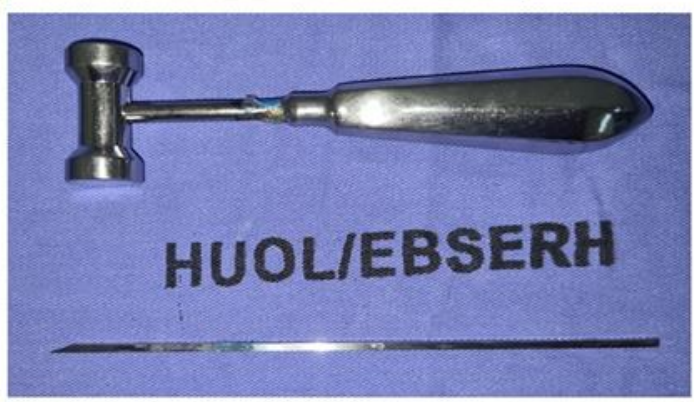

Martelo

Cinzel Reto

Fonte: Unidade de Cabeça e Pescoço HUOL/EBSERH.

Figura 3 - Instrumental Cirúrgico 3

O espéculo de Killian (Figura 03) é o instrumento utilizado para aumentar o campo visual da narina, apresenta-se em diferentes tamanhos de cabeça, sendo ideal ter em sua mesa cirúrgica um longo e outro curto para serem utilizados a depender do estágio cirúrgico.

Na imagem acima temos a descrição do martelo e do cinzel, que são utilizados em nossa técnica habitual de septoplastia, os quais são responsáveis por cortar osso com a colocação da ponta do cinzel na região desejada e neste ponto são dadas leves incursões com o martelo em forma ritmada de dois eventos de choque para uma pausa.

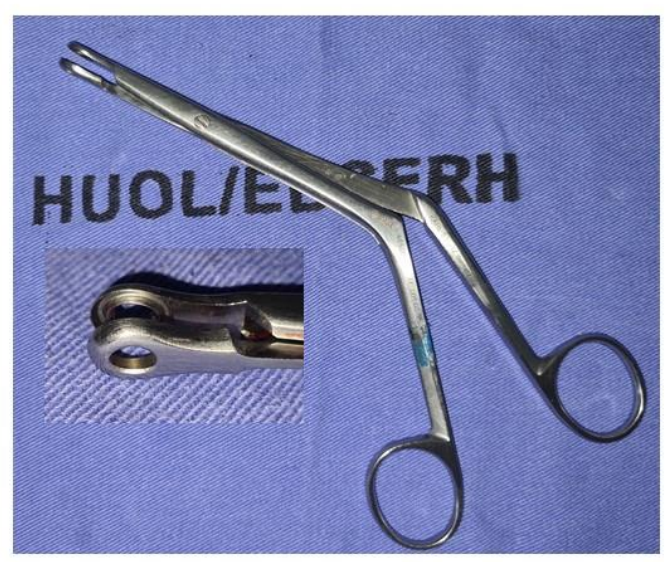

Pinça Bruening

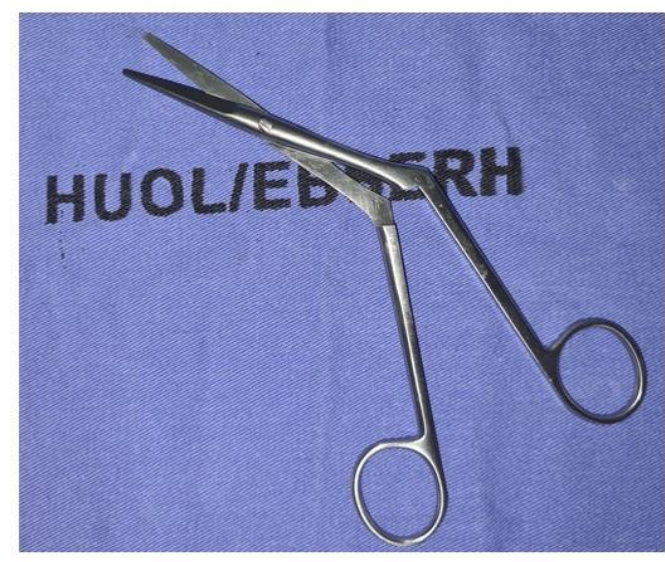

Tesoura Heymann

Fonte: Unidade de Cabeça e Pescoço HUOL/EBSERH.

Figura 4 - Instrumental Cirúrgico 4

A pinça de Bruening (Figura 04) é uma pinça do tipo cortante muito utilizada no processo de retirada de fragmentos de cartilagem e pedaços ósseos. Já a tesoura de 
Instrumentação e montagem de mesa cirúrgica para septoplastia com turbinectomia Bedaque HP, et al

Heymann, também conhecida como tesoura de corneto inferior, é desenvolvida para o procedimento de corte da concha inferior.

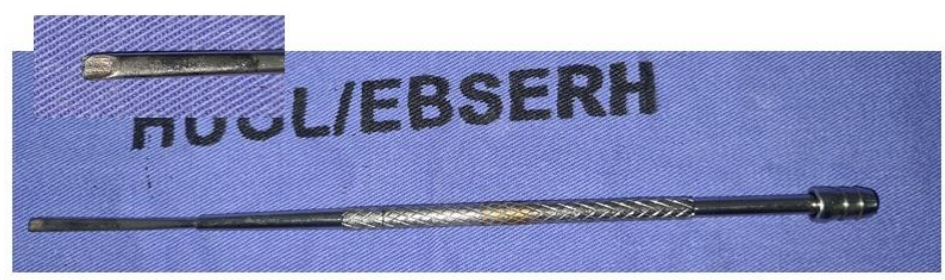

Descolador Aspirador Nasal

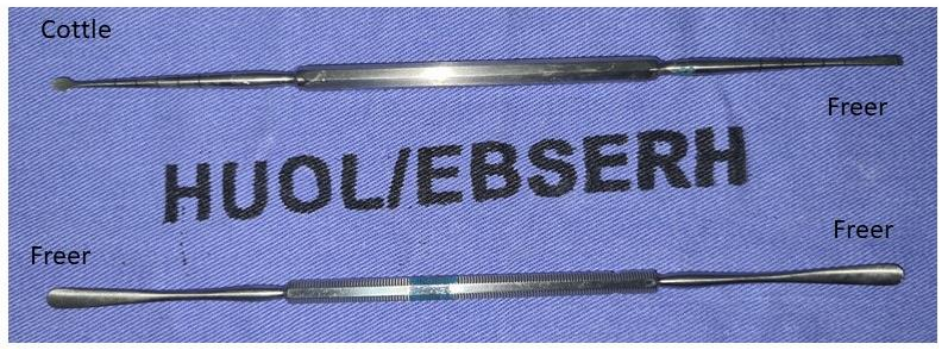

Descolador Cottle

Descolador Freer

Fonte: Unidade de Cabeça e Pescoço HUOL/EBSERH.

Figura 5 - Instrumental Cirúrgico 5

Na figura 05 temos o descolador aspirador nasal que é um dos instrumentos mais importantes nas etapas que sucedem o corte da mucosa nasal anterior, pois o seu manuseio permite que com facilidade seja separada a mucosa nasal do pericôndrio para a posterior retirada da cartilagem desviada.

Além disso, temos o uso dos descoladores que podem ser do tipo Cottle (lembram um triângulo) ou Freer (espátula arredondada) que podem ser utilizados em diferentes momentos cirúrgicos, como na luxação do corneto inferior, na palpação de estruturas anatômicas e no corte de cartilagem.

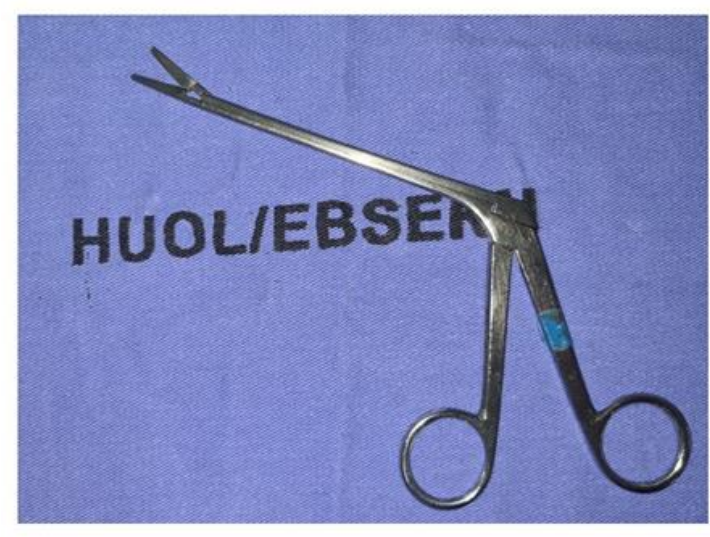

Tesoura de Corneto Médio

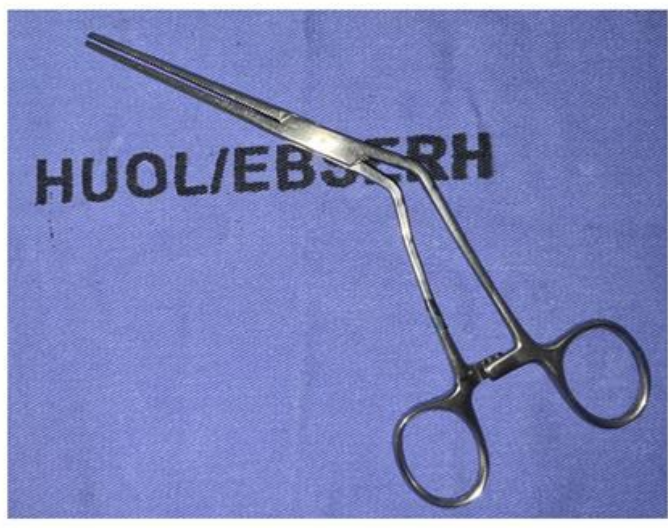

Pinça de Preensão

Fonte: Unidade de Cabeça e Pescoço HUOL/EBSERH.

Figura 6 - Instrumental Cirúrgico 6

A tesoura de corneto médio (Figura 06) tem uma lâmina mais curta do que a tesoura de Heymann, o que a torna mais prática para um eventual corte de concha média ou tratamento de uma concha média bolhosa. Além disso, a pinça de preensão 
Instrumentação e montagem de mesa cirúrgica para septoplastia com turbinectomia Bedaque HP, et al

costuma ser utilizada previamente ao processo de corte da concha inferior, pois ao fazer uma preensão deste com essa pinça é possível deixar uma marca do percurso a ser cortado, além de tornar menor a espessura da concha a ser retirada.

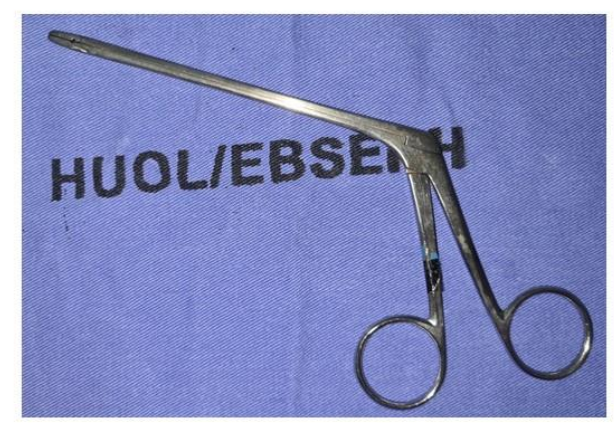

Pinça Takahashi Reta

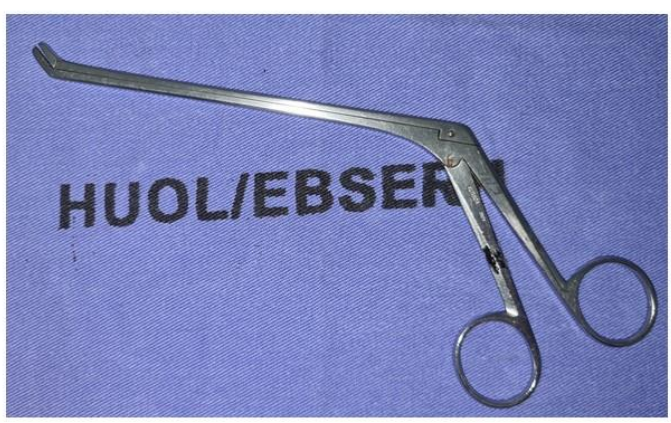

Pinça Takahashi Curva

Fonte: Unidade de Cabeça e Pescoço HUOL/EBSERH.

Figura 7 - Instrumental Cirúrgico 7

Na figura 07 temos a mesma pinça do tipo Takahashi, porém uma em sua apresentação reta e outra curva. São principalmente utilizadas em sua função cortante e para a retirada de fragmentos ósseos e cartilaginosos.

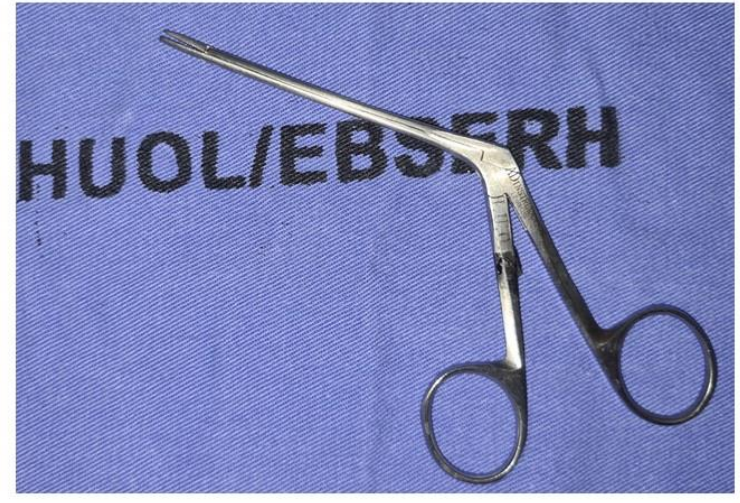

Pinça Hartmann Jacaré

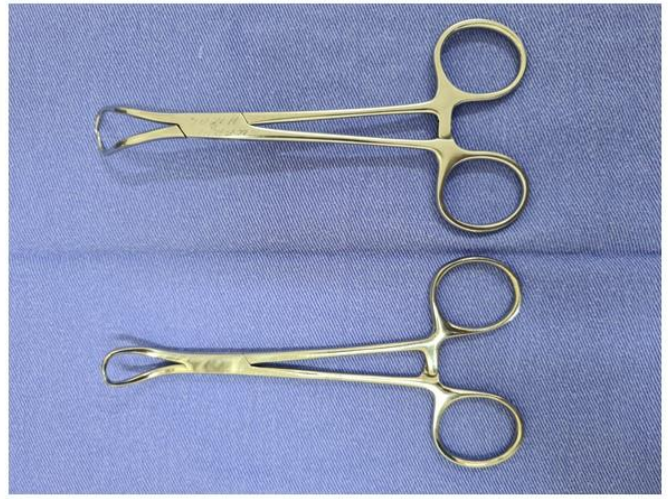

Pinça Backhaus

Fonte: Unidade de Cabeça e Pescoço HUOL/EBSERH.

Figura 8 - Instrumental Cirúrgico 8

A pinça Hartmann Jacaré (Figura 08) é uma pinça coringa para a realização de diferentes etapas, colocação de algodão nos meatos, retirada de fragmentos e eventual realização de tampão nasal. Por fim, a pinça Bachkaus é rotineiramente utilizada para a fixação de outros instrumentos com cabos, para que estes não caiam do campo operatório. 
Instrumentação e montagem de mesa cirúrgica para septoplastia com turbinectomia

Bedaque HP, et al

\section{PREPARAÇÃO DA MESA}

Após o conhecimento sobre os instrumentais disponíveis para o procedimento cirúrgico devemos entender como dispor esses e outros materiais de uso básico em cirurgia na mesa de instrumentos para proporcionar agilidade e organização no desenvolver da cirurgia.

As imagens a seguir representadas tomam como referência um instrumentador de frente para a mesa, desse modo, o lado direito da figura será o mesmo lado direito do instrumentador. Além disso, como padrão para esse tipo de cirurgia, o cirurgião fica à esquerda do paciente e o instrumentador do lado direito do paciente, ao lado do sistema de vídeo.

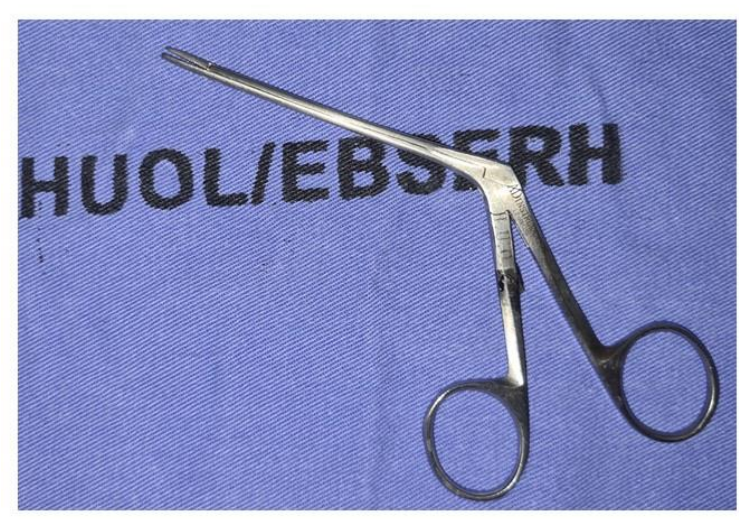

Pinça Hartmann Jacaré

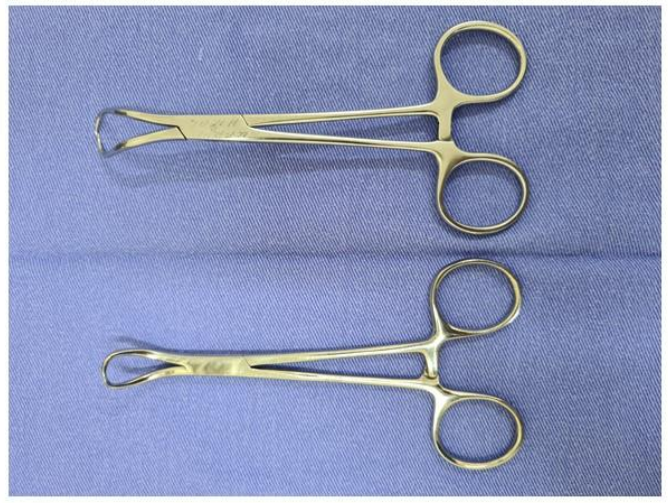

Pinça Backhaus

Fonte: Unidade de Cabeça e Pescoço HUOL/EBSERH.

Figura 9 - Mesa de instrumentação 1

Ao fundo da mesa (Figura 09) organizamos materiais de uso básico em cirurgias, como a compressa e as gazes à direita. Ao centro, uma cuba tipo rim com uma seringa de $20 \mathrm{ml}$ fica em posição estratégica. Após sua colocação, pede-se que um circulante coloque soro fisiológico, preferencialmente gelado, na cuba para poder ser usado, por exemplo, durante a lavagem da cavidade nasal.

Também notamos a presença de dois copos. O primeiro (mais ao fundo) contém uma solução para infiltração de mucosas, uma solução possível é realizada com a adição de $20 \mathrm{ml}$ de água bidestilada, $20 \mathrm{ml}$ de solução padrão de xilocaína sem vaso constritor e $1 \mathrm{ml}$ de adrenalina (solução padrão 1:40.000). O segundo copo é utilizado para embeber retângulos de algodão estéreis que serão utilizados como vasoconstrictores tópicos ao serem colocados dentro da cavidade nasal. A sua solução pode ser obtida com a adição de $5 \mathrm{ml}$ de adrenalina com $5 \mathrm{ml}$ de água bidestilada (solução padrão 1:1000).

Por fim, notamos a caixa de instrumental que pode ficar com peças sobressalentes, como forma de reduzir o risco de contaminação ou de quedas. Ademais, 
Instrumentação e montagem de mesa cirúrgica para septoplastia com turbinectomia Bedaque HP, et al

as Backhaus devem estar facilmente dispostas até a sua colocação nos campos operatórios fixando cabos.

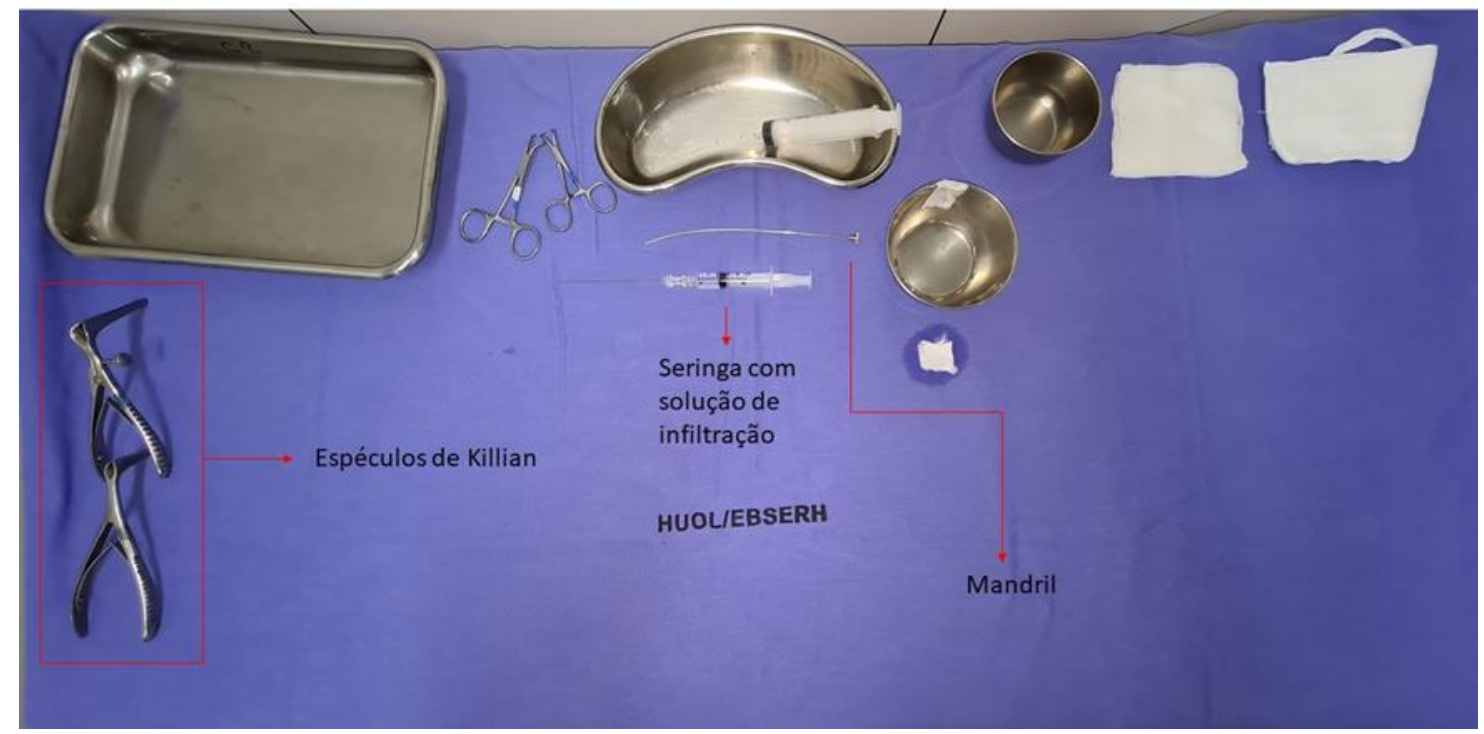

Fonte: Unidade de Cabeça e Pescoço HUOL/EBSERH.

Figura 10 - Mesa de instrumentação 2

Reservamos o canto inferior esquerdo (Figura 10) para a colocação dos espéculos de Killian por trazer uma maior proximidade com o campo operatório, visto que são instrumentos muito utilizados no início e no término do ato cirúrgico. Além disso, é interessante deixar uma seringa de $5 \mathrm{ml}$ preenchida com a solução para infiltração pronta para ser usada e, habitualmente, utilizamos uma agulha de raquianestesia de $\mathrm{N}^{\circ} 22$ acoplada.

Com nome de Mandril o que precisamos é de um material em forma de fio e rígido que possa passar pelo canal de um aspirador nasal ou de um aspirador cautério. O objetivo é que a passagem desse fio de aço consiga desobstruir qualquer material que esteja prejudicado o vácuo do aparelho. Dessa forma, como o procedimento de desobstrução pode ocorrer em qualquer momento, deixamos o instrumento bem posicionado a frente da cuba rim. 
Instrumentação e montagem de mesa cirúrgica para septoplastia com turbinectomia

Bedaque HP, et al

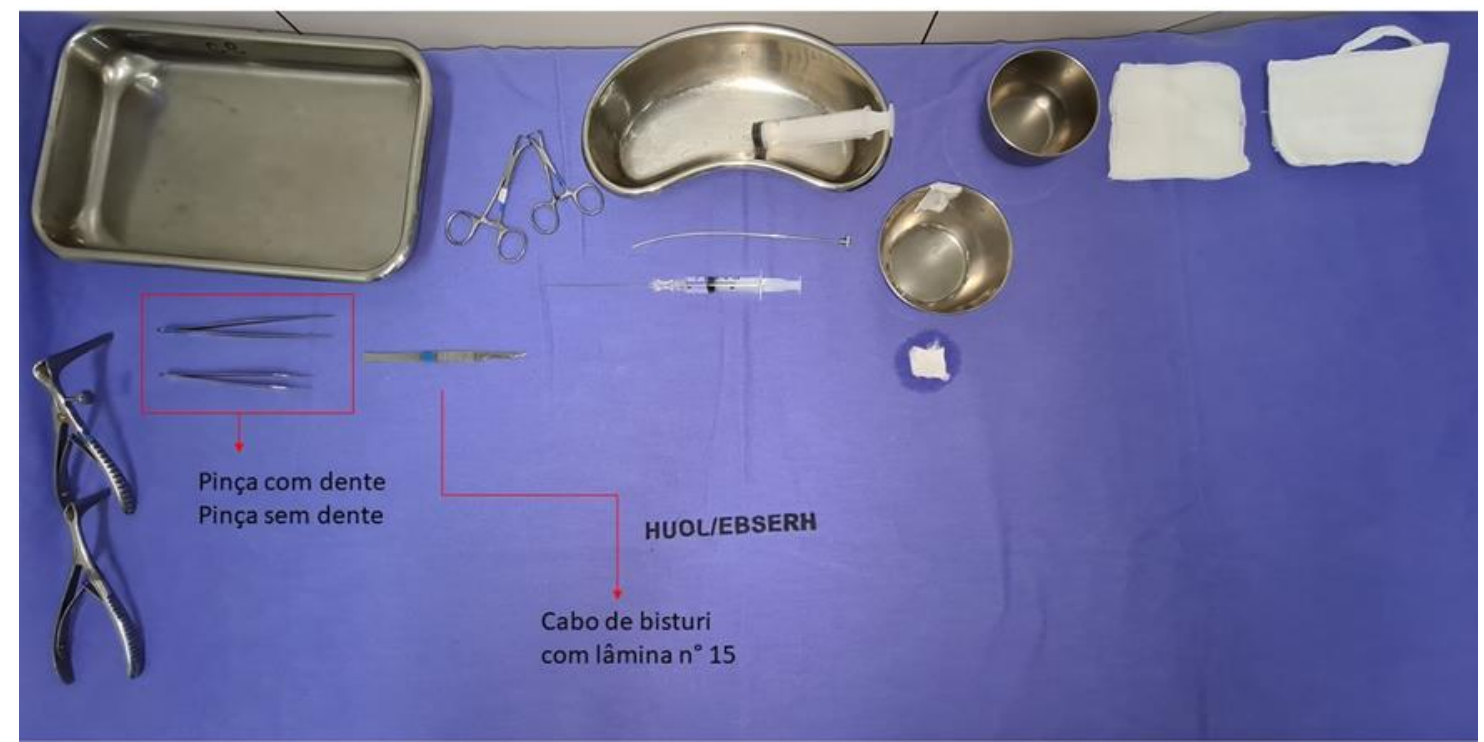

Fonte: Unidade de Cabeça e Pescoço HUOL/EBSERH.

Figura 11 - Mesa de instrumentação 3

Situada à esquerda (Figura 11), abaixo da caixa de instrumental e à direita dos espéculos, colocamos as pinças com e sem dente. As pinças são instrumentos delicados que são muito úteis nas etapas iniciais e finais da septoplastia, de modo que sua proximidade do campo operatório trará benefícios ao fluxo de instrumentação. Além disso, ao lado direito das pinças colocamos em destaque o cabo de bisturi que já deve estar instalado com a lâmina $\mathrm{N}^{\circ} 15$ antes do início do procedimento.

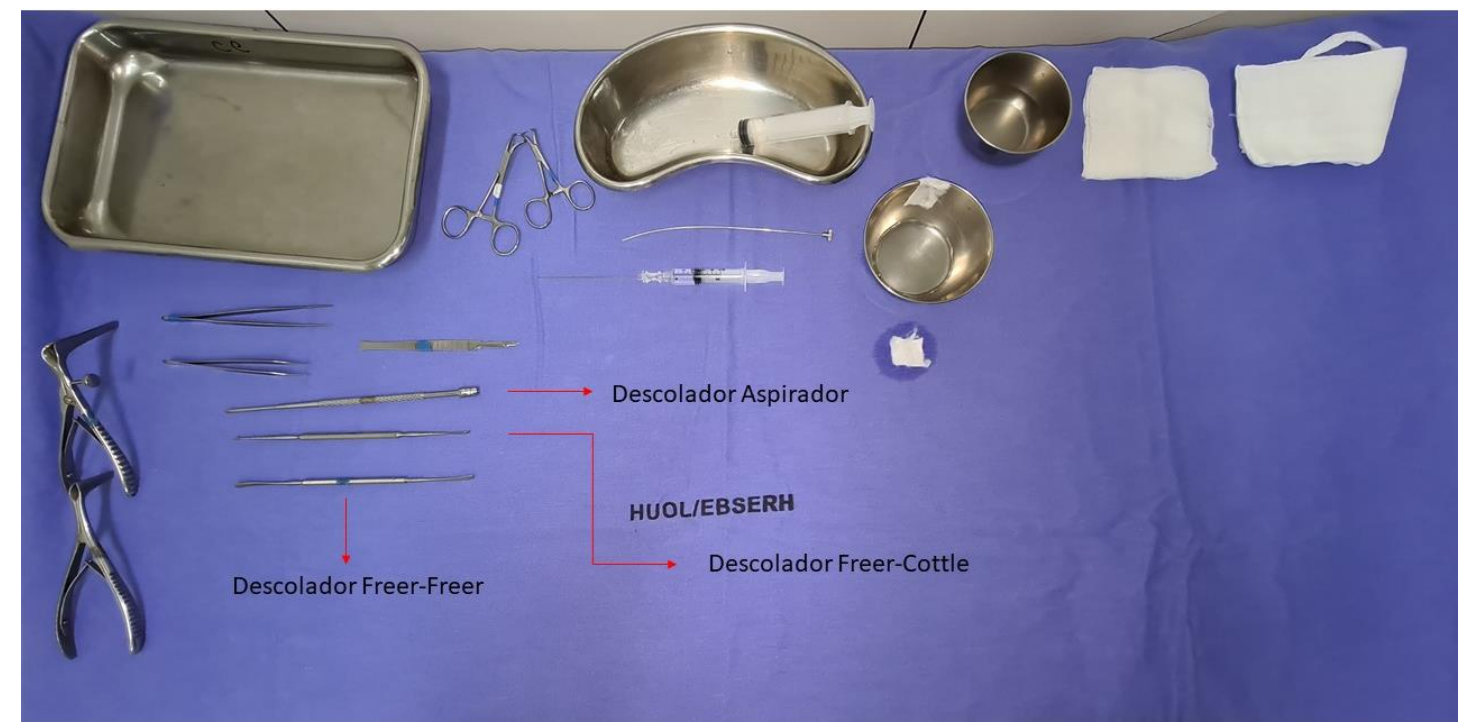

Fonte: Unidade de Cabeça e Pescoço HUOL/EBSERH.

Figura 12 - Mesa de instrumentação 4

Abaixo das pinças e à direita dos espéculos colocamos os instrumentos descoladores (Figura 12). Ao fundo, o descolador aspirador que é mais utilizado no meio do procedimento e este deve estar acoplado a um látex de aspiração, assim, durante o seu uso, deve ficar no campo operatório (lembre-se de fixar o látex com uma pinça Backhaus). 
Instrumentação e montagem de mesa cirúrgica para septoplastia com turbinectomia Bedaque HP, et al

Além disso, colocamos em sequência o descolador Freer-Cottle e descolador Freer-Freer à esquerda, próximo ao campo cirúrgico, exatamente por ser bastante utilizado ao longo do procedimento.

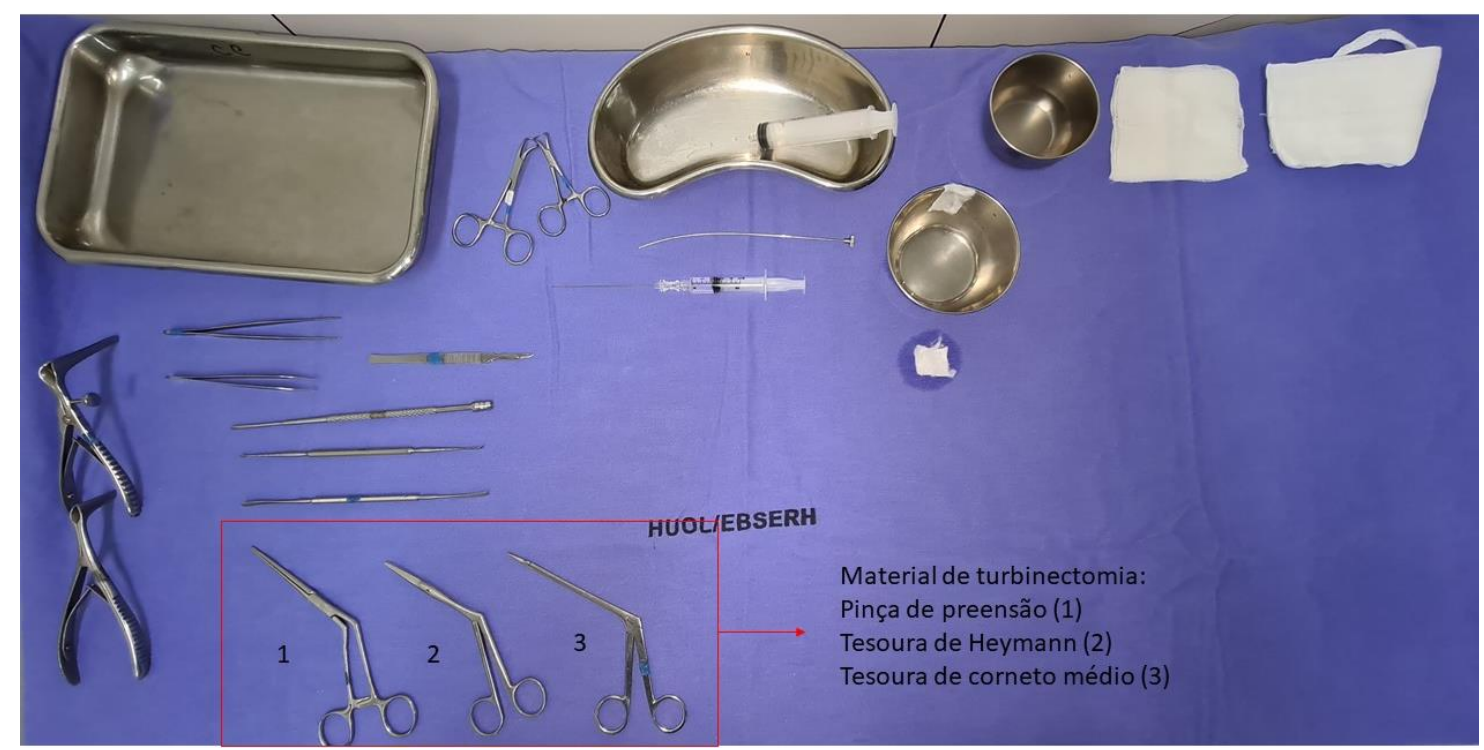

Fonte: Unidade de Cabeça e Pescoço HUOL/EBSERH.

Figura 13 - Mesa de instrumentalização 5

Abaixo dos descoladores e à direita dos espéculos colocamos o material de turbinectomia à disposição (Figura 13). Dessa forma, temos a pinça de preensão (a primeira utilizada na turbinectomia) seguida da tesoura de Heymann e da tesoura de corneto médio (nem sempre utilizada).

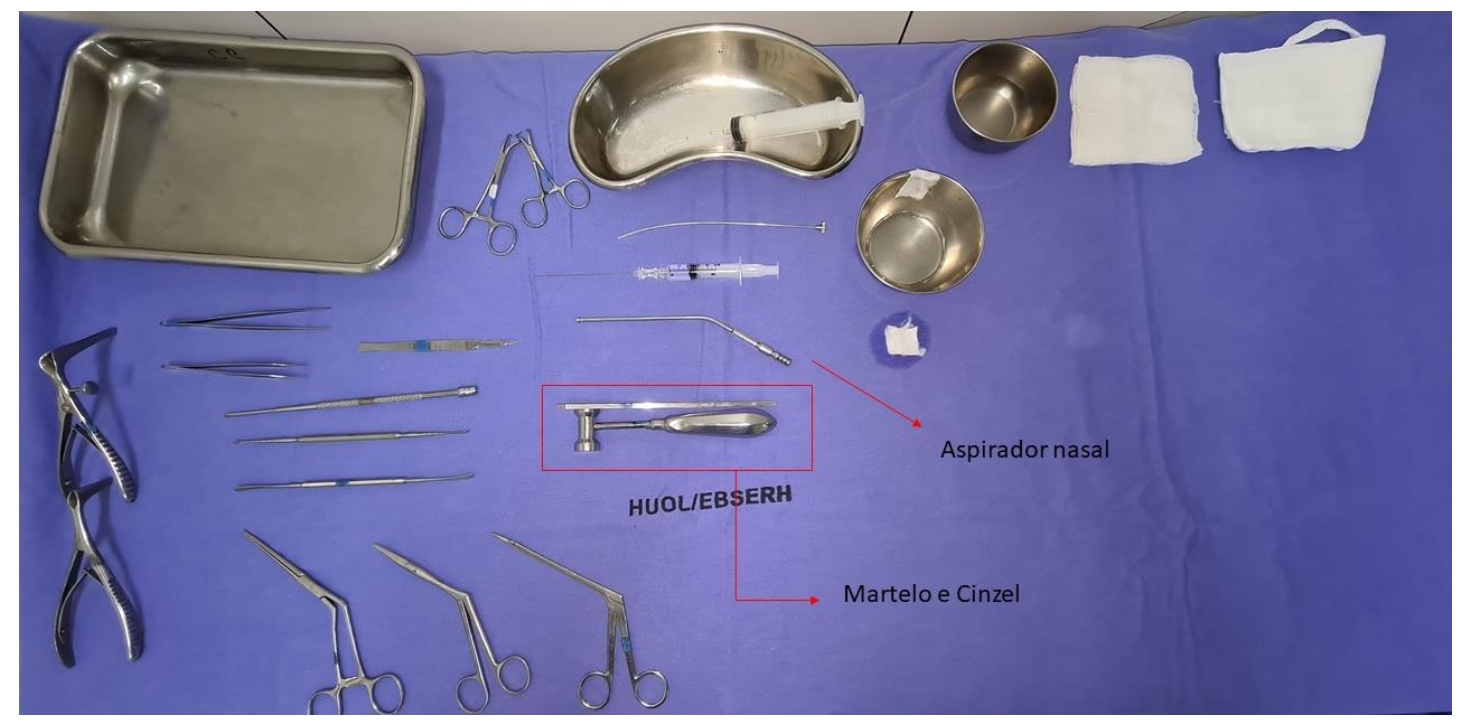

Fonte: Unidade de Cabeça e Pescoço HUOL/EBSERH.

Figura 14 - Mesa de instrumentalização 6

Olhando para o centro da mesa de instrumentos (Figura 14) notamos que embaixo da seringa para infiltração temos o aspirador nasal que permanece na maior parte do ato cirúrgico conectado a um látex e no campo operatório. Entretanto, é interessante estar sempre com um aspirador reserva na mesa de instrumentos 
Instrumentação e montagem de mesa cirúrgica para septoplastia com turbinectomia Bedaque HP, et al

enquanto o outro está em uso no campo, pois caso haja obstrução do aspirador faz-se a troca deste enquanto procede-se à sua desobstrução com o mandril.

Ademais, abaixo do aspirador, bem ao centro da mesa, temos o martelo e o cinzel que serão utilizados costumeiramente no meio das etapas da septoplastia, na etapa de retirada de esporão ósseo, principalmente em região do assoalho nasal.

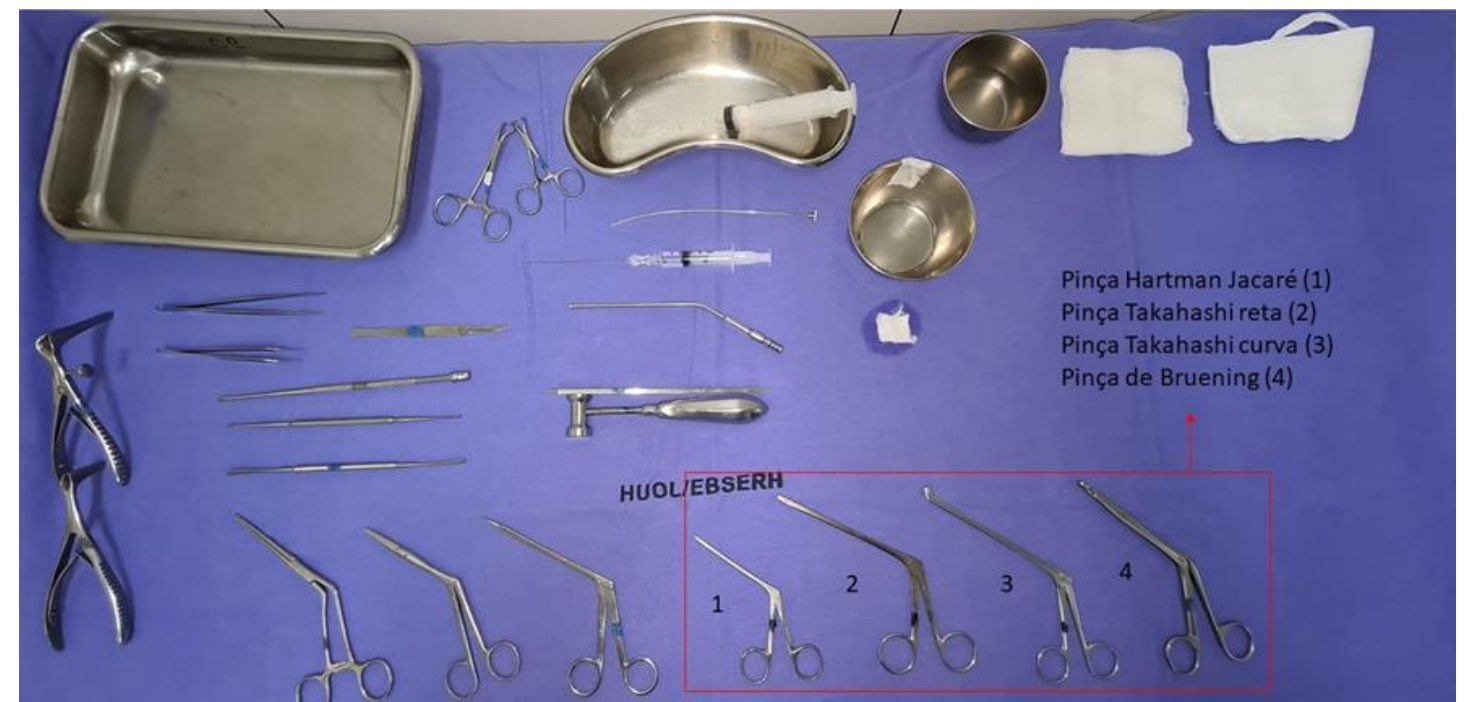

Fonte: Unidade de Cabeça e Pescoço HUOL/EBSERH.

Figura 15 - Mesa de instrumentalização 7

No canto inferior direito (Figura 15) temos as variadas pinças nasais. A pinça Hartman Jacaré com suas variadas funções, como a colocação dos algodões embebidos com solução de adrenalina na cavidade nasal. Em seguida a pinça Takahashi reta e curva que são instrumentos cortantes utilizados para retirada de fragmentos septais. $E$, mais à direta, temos a pinça de Bruening, normalmente utilizada para a retirada de fragmentos septais maiores, por ser mais forte e permitir uma rotação no mesmo eixo de forma firme e segura.

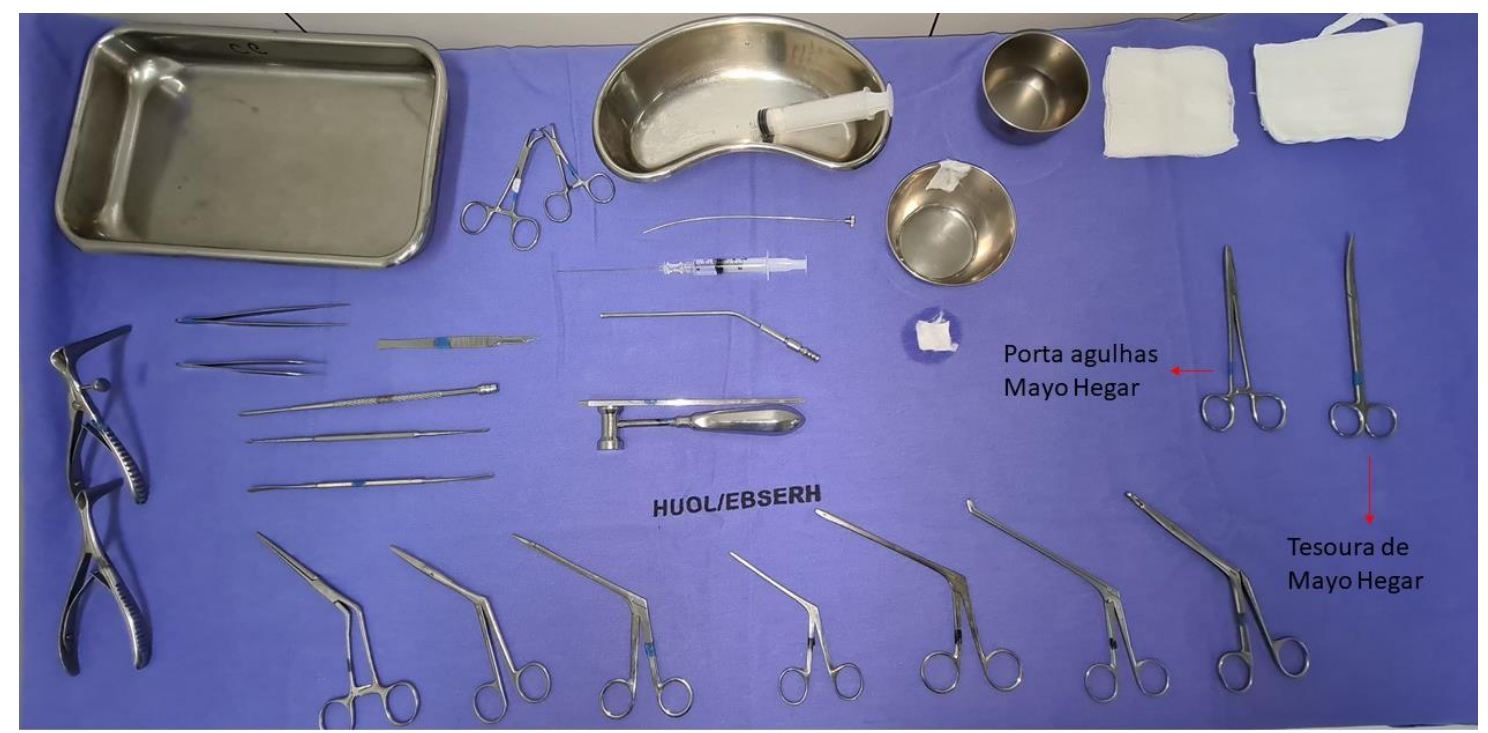

Fonte: Unidade de Cabeça e Pescoço HUOL/EBSERH.

Figura 16 - Mesa de instrumentalização 8 
Instrumentação e montagem de mesa cirúrgica para septoplastia com turbinectomia Bedaque HP, et al

À direta da mesa de instrumentação, mais longe do campo cirúrgico (Figura 16) temos dois instrumentos menos utilizados. O porta agulhas de Mayo Hegar tem sua utilização principal ao final da cirurgia, no procedimento de sutura das mucosas septais e de fechamento da incisão inicial. Já a tesoura é muito utilizada pelo instrumentador para a preparação de diversos materiais, mas para o campo operatório costuma ser utilizado em conjunto com o momento das suturas para o corte dos fios.

\section{OUTRO INSTRUMENTO IMPORTANTE}

Durante a realização de uma septoplastia com turbinectomia existe outro instrumental que não fica na mesa, mas a todo o momento disponível ao longo dos campos cirúrgicos. Como é o caso do aspirador cautério elétrico.

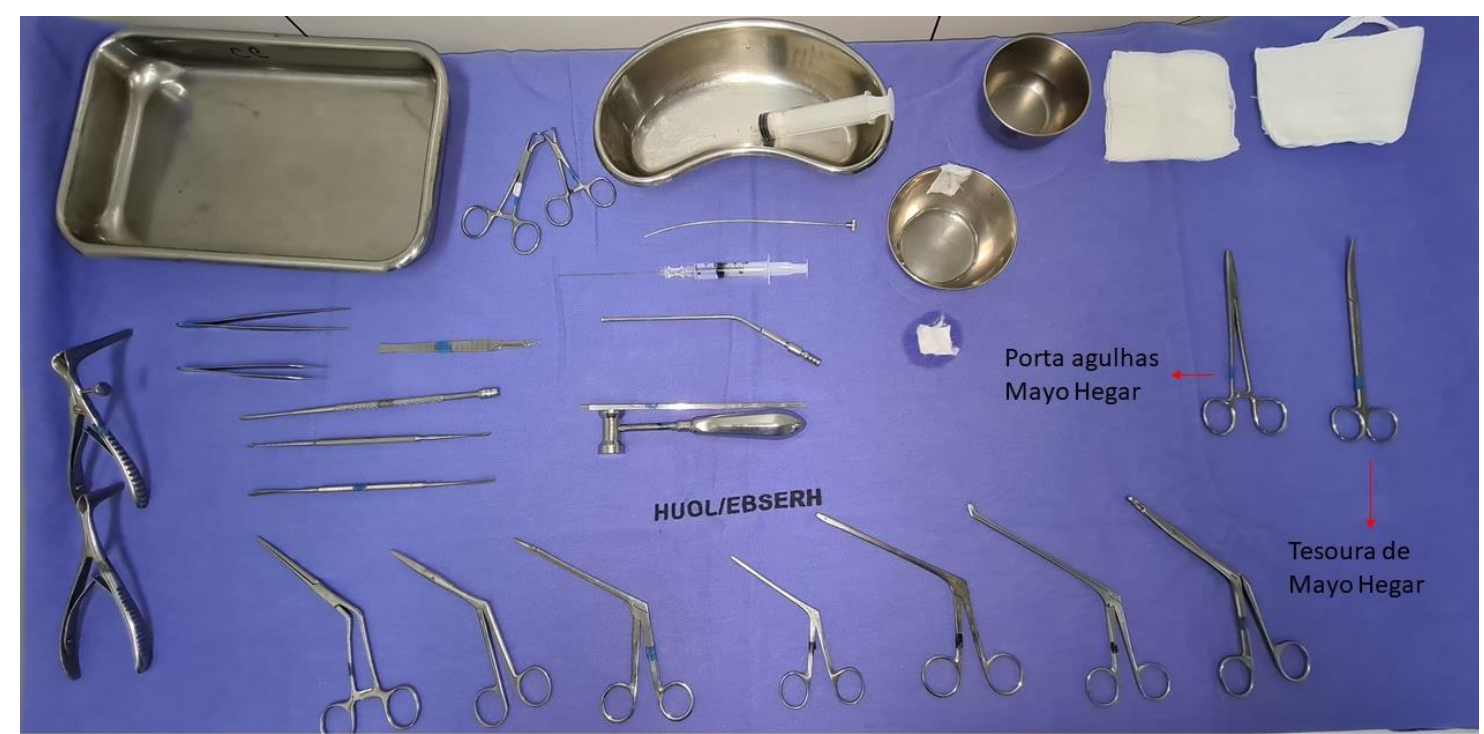

Fonte: Unidade de Cabeça e Pescoço HUOL/EBSERH.

Figura 17 - Aspirador Cautério

O aspirador cautério nasal tem três portas (Figura 17). A primeira, mais à esquerda, é a porta de entrada das secreções aspiradas, a da direita é a região que se conecta com o látex e, consequentemente, ao vácuo (motor de aspiração). Ademais, mais acima temos a porta de entrada do conecto do cautério elétrico. Por fim, notamos um pequeno orifício na região mais à direta que quando obstruído (com um dedo) leva a um aumento da capacidade de aspiração.

\section{CONCLUSÃO}

O conhecimento de boas práticas cirúrgicas é essencial para que o ato operatório ocorra da forma mais segura e eficaz ao paciente. Desse modo, o bom domínio sobre a nomenclatura do material cirúrgico e uma adequada disposição deles na mesa de instrumentais é de grande valia como um facilitador cirúrgico. 
Instrumentação e montagem de mesa cirúrgica para septoplastia com turbinectomia

Bedaque HP, et al

\section{REFERÊNCIAS}

1. Velasco, L. C.; Arima, L. M.; Tiago, R. S. L. Avaliação da melhora dos sintomas nasais após septoplastia com ou sem turbinectomia. Brazilian Journal of Otorhinolaryngology, v. 77, n. 5, p. 577-583, 2011.

2. Most, S. P., \& Rudy, S. F. (2017). Septoplasty: Basic and Advanced Techniques. Facial plastic surgery clinics of North America, 25(2), 161-169. https://doi.org/10.1016/j.fsc.2016.12.002

3. Shah, J.; Roxbury, C. R.; \& Sindwani, R. (2018). Techniques in Septoplasty: Traditional Versus Endoscopic Approaches. Otolaryngologic clinics of North America, 51(5), 909-917.

4. Bezerra, T. F. P.; et al. Avaliação da qualidade de vida após septoplastia em pacientes com obstrução nasal. Brazilian Journal of Otorhinolaryngology, v. 78, n. 3, p. 57-62, 2012.

5. Gobbo, T. F.; et al. Estudio retrospectivo de 1692 casos de turbinectomías, septoplastias y polipectomías por microcirugía endonasal. Rev Bras Otorrinolaringol, v. 61, n. 2, p. 114-20, 1995.

6. Patrocínio, L. G.; et al. Perfil do atendimento otorrinolaringológico em clínica privada. Arq Int Otorrinolaringol, v. 11, n. 2, p. 130-34, 2007.

7. Getz, A. E., \& Hwang, P. H. (2008). Endoscopic septoplasty. Current opinion in otolaryngology \& head and neck surgery, 16(1), 26-31.

8. Organização Mundial da Saúde. Segundo desafio global para a segurança do paciente: Manual - cirurgia segura salvam vidas; Tradução de Marcela Sanches Nilo e Irma Angégila Durán. Rio de Janeiro: Organização Pan-Americana de Saúde; Ministério da Saúde; Agência Nacional de Vigilância Sanitária, 2009. ISBN: 978-8587943-98-9.

9. Yoon, S.; Zygourakis, C. C.; Seaman, J.; Zhu, M.; Ahmed, A. K.; Kliot, T.; Antrum, S.; Goldberg, A. N. Implementation and Impact of a Hospital-Wide Instrument Set Review: Early Experiences at a Multisite Tertiary Care Academic Institution. Am J Med Qual. 2019 Jan/Feb;34(1):67-73. doi: 10.1177/1062860618783261. Epub 2018 Jun 25. PMID: 29936862. 